\title{
AN EXTENSION OF PÓLYA'S THEOREM ON POWER SERIES WITH INTEGER COEFFICIENTS
}

\author{
BY \\ RAPHAEL M. ROBINSON
}

1. Introduction. The theorem which we shall extend is the following.

Pólya's THEOREM. Let $E$ be a bounded closed set which is symmetric to the real axis. Suppose that the complement of $E$ is a region $G$. Suppose that $f(z)$ is regular in $G$ and near $\infty$ has the expansion

$$
f(z)=\sum_{v=0}^{\infty} a_{v} z^{-v}
$$

with integer coefficients. If the transfinite diameter of $E$ is less than 1 , then $f(z)$ must be rational.

This was proved first by Pólya [4] for the case in which $G$ is simply connected. A general proof was given later by Pólya [5, p. 703]. (It is understood that $f(z)$ is single valued in $G$.)

A more precise conclusion is that $f(z)$ can be expressed in the form $P(z) / Q(z)$, where $P(z)$ and $Q(z)$ are relatively prime polynomials with integer coefficients, and $Q(z)$ has its leading coefficient equal to 1 . Thus the poles of $f(z)$ can occur only at sets of conjugate algebraic integers lying in $E$. By a theorem of Fekete [2], there are only a finite number of such algebraic integers when the transfinite diameter of $E$ is less than 1.

Pólya's theorem is an extension of a theorem of Carlson [1], according to which a function $f(z)$ of the same form which is regular for $|z|>1$ must either be rational or have the unit circle as a natural boundary. (This was stated originally for power series regular inside the unit circle.)

In [4], Pólya also proved the converse for the case in which $G$ is simply connected. That is, if the transfinite diameter of $E$ is at least 1 , then there exist nonrational functions $f(z)$ whose expansions at $\infty$ have integer coefficients; indeed, there are a nondenumerable infinity of such functions. I have not seen a proof of the converse in general. One will be given in this paper as part of our discussion of the extended Pólya theorem.

Fekete [2] proved that the transfinite diameter of $E$ is less than 1 if and only if there exists a polynomial

$$
h(z)=z^{r}+A_{r-1} z^{r-1}+\cdots+A_{0}
$$

such that $|h(z)|<1$ on $E$. We may take the coefficients to be real. Indeed, using a

Presented to the Society, December 29, 1965; received by the editors December 1, 1966. 
method of Kakeya, we may even take them to be integers; see Fekete and Szegö $[3, \S 2]$. Thus the condition in Pólya's theorem that the transfinite diameter of $E$ is less than 1 may be replaced by the existence of such a function $h(z)$, where we may suppose either that the coefficients are real or that they are integers.

We now raise the question whether there is a similar result about a function $f(z)$ which has expansions with integer coefficients at several points. The simplest case is the following. Suppose that $G$ contains 0 as well as $\infty$, and that

$$
f(z)=\sum_{v=0}^{\infty} a_{v} z^{-v} \text { near } \infty, \quad f(z)=\sum_{v=0}^{\infty} b_{v} z^{v} \text { near } 0,
$$

where both series have integer cofficients. We can conclude that $f(z)$ is rational if there exists a function

$$
h(z)=z^{r}+A_{r-1} z^{r-1}+\cdots+A_{-s+1} z^{-s+1}+z^{-s},
$$

with $r>0$ and $s>0$, such that $|h(z)|<1$ on $E$. Indeed, the conclusion can be drawn only in this case. If there is any such function $h(z)$, then there is also one with integer coefficients. If $h(z)$ has integer coefficients, then the poles of $f(z)$ occur at zeros of $h(z)$, and hence lie at sets of conjugate algebraic units contained in $E$.

As compared to Pólya's theorem, this result obtains the conclusion that $f(z)$ is rational by allowing more singularities for $f(z)$ at the expense of requiring additional information concerning its series expansions.

The general result which we shall prove is the following.

MAIN TheORem. Let $p_{1}, \ldots, p_{l}, q_{1}, \ldots, q_{l}$ be integers such that $q_{k} \neq 0$ and $p_{j}-p_{k} \mid q_{k}$ for $j \neq k$. Let $E$ be a bounded closed set symmetric to the real axis and not containing any of the points $p_{1}, \ldots, p_{l}$. Let the complement of $E$ be a region $G$. Suppose that $f(z)$ is regular in $G$ and has expansions

$$
\begin{aligned}
& f(z)=\sum_{v=0}^{\infty} a_{v} z^{-v} \text { near } \infty, \\
& f(z)=\sum_{v=0}^{\infty} b_{k v}\left(\frac{z-p_{k}}{q_{k}}\right)^{v} \text { near } p_{k} \quad(k=1,2, \ldots, l),
\end{aligned}
$$

where the $a_{v}$ and $b_{k v}$ are integers. If, for some $r>0, s_{1}>0, \ldots, s_{l}>0$, there is a function

$$
h(z)=\sum_{\nu=0}^{r} A_{v} z^{v}+\sum_{k=1}^{l} \sum_{\nu=1}^{s_{k}} B_{k v}\left(\frac{q_{k}}{z-p_{k}}\right)^{v}
$$

with

$$
\left|A_{r}\right| \geqq 1, \quad\left|B_{k s_{k}}\right| \geqq 1 \text { for } k=1,2, \ldots, l,
$$

such that $|h(z)|<1$ on $E$, then $f(z)$ is rational.

According to [6, Theorem 3.1], if there is any $h(z)$ with the required properties, 
then there is also one with integer coefficients. Furthermore, the leading coefficients may all be taken equal to 1 . Thus it will be sufficient to prove the main theorem under the assumption that the $A_{v}$ and $B_{k v}$ are integers. This will be done in $\$ 2$. In this case, we will find that the poles of $f(z)$ are zeros of $h(z)$. Taking the leading coefficients of $h(z)$ equal to 1 , and applying [6, Theorem 6.1], we find that the poles of $f(z)$ must occur at sets of conjugate algebraic integers $\theta$ lying in $E$ and satisfying the conditions $\theta-p_{k} \mid q_{k}$ for $k=1,2, \ldots, l$.

It is shown in $[6, \S 2]$, that the conditions on $p_{1}, \ldots, p_{l}, q_{1}, \ldots, q_{l}$ are preserved by the linear transformation $w=q_{1} /\left(z-p_{1}\right)$, which takes $p_{1}$ to $\infty$. Thus $\infty$ plays the same role as the points $p_{1}, \ldots, p_{l}$. In particular, it could be omitted as one of the points considered in the main theorem. Simply delete the condition that $E$ is bounded and the assumption concerning the form of $f(z)$ near $\infty$, and replace the term $\sum A_{v} z^{v}$ in the formula for $h(z)$ by $A_{0}$.

It may be noted that, when the proof given in $\S 2$ is specialized to the case $l=0$ we obtain an entirely new proof of Pólya's theorem itself.

In $\S 3$, we note an unexpected result which turns up when we apply a similar method even though the set $G$ is not connected.

We then turn to the converse. After some preparation in $\$ 4$, we prove in $\$ 5$ that if there is no $h(z)$ of the form required in the main theorem with $|h(z)|<1$ on $E$, then there exist nonrational functions $f(z)$ with expansions of the required sort which are regular in $G$, and indeed there are nondenumerably many such functions.

2. Proof of the main theorem. We shall now prove the main theorem under the assumption that the coefficients of $h(z)$ are integers. We start with some comments about the divisibility conditions $p_{j}-p_{k} \mid q_{k}$. As noted in [6, §2], these guarantee that the functions $h(z)$ with integer coefficients form a set closed under multiplication. Furthermore, it follows that the Laurent expansion of $h(z)$ has integer coefficients at $\infty$, and at $p_{k}$ it has integer coefficients when expanded in powers of $\left(z-p_{k}\right) / q_{k}$. To see this, notice that at $\infty$ we have

$$
\frac{q}{z-p}=\frac{q}{z} \sum_{v=0}^{\infty} \frac{p^{v}}{z^{v}}
$$

and at $p$ we have

$$
\begin{aligned}
z & =p+q \cdot \frac{z-p}{q} \\
\frac{q^{\prime}}{z-p^{\prime}} & =\frac{q^{\prime}}{p-p^{\prime}} \sum_{\nu=0}^{\infty}\left(\frac{q}{p^{\prime}-p}\right)^{\nu}\left(\frac{z-p}{q}\right)^{v} .
\end{aligned}
$$

Only in the last case do we need the divisibility conditions.

We shall show that $f(z)$ is rational by studing $f(z) h(z)^{n}$ for large $n$. This will be proved to be rational, with poles at $\infty, p_{1}, \ldots, p_{l}$ only. Hence $f(z)$ itself will be rational, but its poles can occur only at zeros of $h(z)$. 
Choose $\lambda<1$ so that $|h(z)|<\lambda$ on $E$. Let $C$ be the curve (which may consist of several loops) on which $|h(z)|=\lambda$. Then $C$ encloses $E$. Let $R(z)$ be any function of the form permitted for $h(z)$ and having integer coefficients. Consider the integral

$$
\frac{1}{2 \pi i} \int_{C} f(z) h(z)^{n} R(z) d z .
$$

This is an integer, since it is equal to minus the sum of the residues at $\infty$ and the various $p_{k}$. At $\infty$, all of the coefficients are integers. At $p_{k}$, we have integer coefficients times powers of $\left(z-p_{k}\right) / q_{k}$, so the coefficient of $\left(z-p_{k}\right)^{-1}$ is an integer.

For any given $R(z)$, the absolute value of the integral is at most $K \lambda^{n}$, where $K$ is a constant, and hence is less than 1 for $n$ sufficiently large. This means that the integral is 0 for $n$ sufficiently large. Now consider any $R(z)$ with poles of orders no larger than for $h(z)$. The integral of each term is 0 for $n$ sufficiently large, say $n \geqq N$. Hence the integral is 0 for all such $R(z)$, whether the coefficients are integers or not.

Finally, we shall show that the integral is always 0 for $n=N$, regardless of the orders of the poles of $R(z)$. If possible, choose the smallest positive integers $r^{\prime}$, $s_{k}^{\prime}$ so that

$$
\begin{gathered}
\int_{C} f(z) h(z)^{N} z^{r^{\prime}} d z \neq 0, \\
\int_{C} f(z) h(z)^{N}\left(\frac{q_{k}}{z-p_{k}}\right)^{s_{k}^{\prime}} d z \neq 0 \quad(k=1,2, \ldots, l) .
\end{gathered}
$$

If impossible, put $r^{\prime}=\infty$ or $s_{k}^{\prime}=\infty$. We want to show that $r^{\prime}=s_{1}^{\prime}=\cdots=s_{l}^{\prime}=\infty$.

By hypothesis, $r^{\prime}>r$ and $s_{k}^{\prime}>s_{k}$. If not all of the numbers $r^{\prime}, s_{1}^{\prime}, \ldots, s_{l}^{\prime}$ are infinite, choose the largest positive integer $\kappa$ such that

$$
\kappa r<r^{\prime}, \quad \kappa s_{k}<s_{k}^{\prime} \quad(k=1,2, \ldots, l) .
$$

Suppose, for example, that $r^{\prime} \leqq(\kappa+1) r$. Now $h(z)^{\kappa}$ has poles of orders $\kappa r, \kappa s_{1}, \ldots$, $\kappa s_{l}$ at $\infty, p_{1}, \ldots, p_{l}$. For the product $h(z)^{\kappa} z^{r^{\prime}-\kappa r}$, the pole at $\infty$ is of order $r^{\prime}$; the order of the pole at $p_{k}$ is not increased, hence is $\leqq \kappa s_{k}<s_{k}^{\prime}$. Thus

$$
\int_{C} f(z) h(z)^{N} h(z)^{\kappa} z^{r^{\prime}-\kappa r} d z \neq 0,
$$

since there is exactly one nonzero term. On the other hand, the integrand has the form $f(z) h(z)^{n} z^{m}$ with $n>N$ and $m \leqq r$, and hence the integral is 0 by the definition of $N$. A similar contradiction is obtained if $s_{k}^{\prime} \leqq(\kappa+1) s_{k}$, by looking at the integral

$$
\int_{C} f(z) h(z)^{N} h(z)^{\kappa}\left(\frac{q_{k}}{z-p_{k}}\right)^{s_{k}^{\prime}-\kappa s_{k}} d z
$$

Thus we must have $r^{\prime}=s_{1}^{\prime}=\cdots=s_{l}^{\prime}=\infty$. That is,

$$
\int_{C} f(z) h(z)^{N} R(z) d z=0
$$


for all rational functions $R(z)$ having poles only at $\infty, p_{1}, \ldots, p_{l}$, and in particular for polynomials.

We can now apply this result to obtain the desired conclusion. We have

$$
\frac{1}{2 \pi i} \int_{C} f(z) h(z)^{N} \prod_{k=1}^{l}\left(\frac{z-p_{k}}{q_{k}}\right)^{N s_{k}} z^{m-1} d z=0
$$

for all $m \geqq 1$. Since the integrand is regular at all the points $p_{k}$, we may replace the curve $C$ by a large circle. If we put

$$
f(z) h(z)^{N} \prod_{k=1}^{l}\left(\frac{z-p_{k}}{q_{k}}\right)^{N s_{k}}=\sum_{\nu=-\infty}^{N r+N s_{1}+\cdots+N s_{l}} c_{\nu} z^{\nu}
$$

near $\infty$, then the above integral is $c_{-m}$. Hence $c_{-m}=0$ for $m \geqq 1$, so the right side is a polynomial. Thus $f(z) h(z)^{N}$ is a rational function whose poles lie at $\infty, p_{1}, \ldots$, $p_{l}$, and $f(z)$ itself is a rational function whose poles lie at the zeros of $h(z)$.

3. A by-product. In the main theorem, we supposed that the complement of $E$ is a region $G$. If we drop this assumption, then $G$ might split into several regions. In particular, some of the points $p_{k}$ might not lie in the same region with $\infty$, so that the various expansions of $f(z)$ would really be expansions of different functions. Thus we have a quite unreasonable hypothesis. However, it is of interest to see what happens to the proof in this case. The results of this section are not used in the rest of the paper, hence the reader may proceed to $\$ 4$ if he prefers.

Suppose first that $h(z)$ has integer coefficients. As before, letting $C$ denote the curve on which $|h(z)|=\lambda$, we find that

$$
\int_{C} f(z) h(z)^{N} R(z) d z=0
$$

for any rational function $R(z)$ having poles only at $\infty, p_{1}, \ldots, p_{l}$. Apply this result with $f(z)=1$ in the region containing $\infty$ and $f(z)=0$ elsewhere. Letting $C^{\prime}$ be the portion of $C$ in the region containing $\infty$, we have

$$
\int_{C^{\prime}} h(z)^{N} R(z) d z=0 .
$$

Suppose that the points $p_{1}, \ldots, p_{l}$ are numbered so that the ones not separated from $\infty$ by $E$ are $p_{1}, \ldots, p_{l^{\prime}}$. Then

$$
\frac{1}{2 \pi i} \int_{C^{\prime}} h(z)^{N} \prod_{k=1}^{l^{\prime}}\left(\frac{z-p_{k}}{q_{k}}\right)^{N s_{k}} z^{m-1} d z=0
$$

for $m \geqq 1$. Since the integrand is regular at $p_{1}, \ldots, p_{l^{\prime}}$, we can replace $C^{\prime}$ by a large circle. If we put

$$
h(z)^{N} \prod_{k=1}^{l^{\prime}}\left(\frac{z-p_{k}}{q_{k}}\right)^{N s_{k}}=\sum_{\nu=-\infty}^{N r+N s_{1}+\cdots+N s_{l^{\prime}}} c_{\nu}^{\prime} z^{\nu}
$$


near $\infty$, then the above integral is $c_{-m}^{\prime}$. Hence $c_{-m}^{\prime}=0$ for $m \geqq 1$, so the right side is a polynomial. Thus $h(z)$ has no poles except $\infty, p_{1}, \ldots, p_{l^{\prime}}$. It follows that $l^{\prime}=l$, and so $E$ does not separate any of the points $p_{k}$ from $\infty$.

Suppose that we have any function

$$
h(z)=\sum_{v=0}^{r} A_{v} z^{v}+\sum_{k=1}^{l} \sum_{v=1}^{s_{k}} B_{k v}\left(\frac{q_{k}}{z-p_{k}}\right)^{v}
$$

with $r>0, s_{1}>0, \ldots, s_{l}>0$, and

$$
\left|A_{r}\right| \geqq 1, \quad\left|B_{k s_{k}}\right| \geqq 1 \text { for } k=1,2, \ldots, l,
$$

such that $|h(z)|<1$ on $E$. By [6, Theorem 3.1], there is also such a function with integer coefficients. It follows that $E$ cannot separate any of the points $p_{k}$ from $\infty$. In other words, if $E$ separates any of the points $p_{k}$ from $\infty$, then there is no function $h(z)$ of the above form such that $|h(z)|<1$ on $E$.

We obtain this result only under arithmetical conditions on the numbers $p_{k}$ and $q_{k}$ which appear quite irrelevant here. However, I do not know what the proper formulation of the theorem is, or how it should be proved.

In the simplest case, we have the following result: Let

$$
h(z)=z^{r}+A_{r-1} z^{r-1}+\cdots+A_{-s+1} z^{-s+1}+z^{-s},
$$

where $r>0$ and $s>0$. Then we cannot have $|h(z)|<1$ on a bounded closed set $E$ which separates 0 and $\infty$. In other words, if $R<1$, then the set $|h(z)| \leqq R$ does not separate 0 and $\infty$.

For any such function $h(z)$, there will be a smallest value of $R$ such that $|h(z)| \leqq R$ separates 0 and $\infty$. Call this value $R_{0}$. We have just shown that $R_{0} \geqq 1$. It appears probable that we always have $R_{0}>1$, that is, that we cannot have $|h(z)| \leqq 1$ on a set $E$ separating 0 and $\infty$.

Added in proof (July 1967). Using a suggestion of M. M. Schiffer, this may be proved as follows. Let $C$ be a simple closed curve separating 0 and $\infty$, and denote by $M$ the maximum of $|h(z)|$ on $C$. Let $\rho_{0}$ be the inner radius of $C$ with respect to the origin, and let $\rho_{\infty}$ be the outer radius of $C$. Then we can map $|w|<\rho_{0}$ one-to-one and conformally onto the interior of $C$ by a function $z=\phi(w)$ with $\phi(0)=0$ and $\phi^{\prime}(0)=1$, and we can map $|w|>\rho_{\infty}$ one-to-one and conformally onto the exterior of $C$ by a function $z=\psi(w)$ with $\psi(\infty)=\infty$ and $\psi^{\prime}(\infty)=1$. It is known that $\rho_{0} \leqq \rho_{\infty}$, with equality only when $C$ is a circle about the origin; see, for example, G. Pólya and G. Szegö, Aufgaben und Lehrsätze aus der Analysis, Vol. 2, IV, problem 127.

The function $h(\phi(w))$ is regular for $|w|<\rho_{0}$ except for a pole at 0 , where the leading term is $w^{-s}$; similarly, $h(\psi(w))$ is regular for $|w|>\rho_{\infty}$ except for a pole at $\infty$, where the leading term is $w^{r}$. Both functions are continuous up to the boundary, and have absolute value at most $M$ there. Cauchy's estimate for the coefficients of the leading terms yields $M \geqq \rho_{0}^{-s}$ and $M \geqq \rho_{\infty}^{r}$. If $C$ is not a circle about the origin, then $\rho_{0}<\rho_{\infty}$ and hence $M>1$. If $C$ is the circle $|z|=\rho$, then Cauchy's estimate 
may be applied directly to $h(z)$, yielding $M>\rho^{-s}$ and $M>\rho^{r}$, with strict inequality since $h(z)$ is not a monomial; hence $M>1$ is this case also. Thus the set $|h(z)| \leqq 1$ cannot separate 0 and $\infty$. [End of added matter.]

The curve $|h(z)|=R_{0}$ must have a double point. Hence there exists a point $z_{0}$ where $h^{\prime}\left(z_{0}\right)=0$ and $\left|h\left(z_{0}\right)\right|=R_{0}$. This may be used to compute $R_{0}$ in some cases. For example, consider the function $h(z)=z^{r}+z^{-s}$. Here the roots of $h^{\prime}(z)=0$ satisfy the equation $z^{r+s}=s / r$. At all of these points, $|h(z)|$ has the same value, which must be $R_{0}$. From this, we find that

$$
R_{0}=1 /\left[\sigma^{\sigma}(1-\sigma)^{1-\sigma}\right], \text { where } \sigma=s /(r+s) .
$$

Thus $R_{0} \rightarrow 1$ as $\sigma \rightarrow 0$ or $\sigma \rightarrow 1$. Hence there exist functions $h(z)$ for which $R_{0}$ is as near to 1 as we please.

4. Preparation for the converse. The purpose of this section is to prove the following theorem, which will be used in $\S 5$ in the proof of the converse of the main theorem.

THEOREM. Let $p_{1}, \ldots, p_{l}, q_{1}, \ldots, q_{l}$ be real numbers with $p_{j} \neq p_{k}$ for $j \neq k$ and $q_{k} \neq 0$. Let $E$ be a bounded closed set which is symmetric to the real axis and does not contain any of the points $p_{k}$. Suppose that the complement of $E$ is a region $G$, and let $F$ be any closed subset of $G$. Then there is a function

$$
H(z)=\sum_{v=0}^{r} A_{v} z^{v}+\sum_{k=1}^{l} \sum_{v=1}^{s_{k}} B_{k v}\left(\frac{q_{k}}{z-p_{k}}\right)^{v}
$$

with real coefficients, $r>0$ and $A_{r} \neq 0, s_{k}>0$ and $B_{k s_{k}} \neq 0$ for $k=1,2, \ldots, l$, and having all of its zeros in $E$, such that $|H(z)|<1$ on $E$ and $|H(z)|>1$ on $F$. We may suppose that either

$$
\left|A_{r}\right|<1, \quad\left|B_{k s_{k}}\right|<1 \text { for } k=1,2, \ldots, l,
$$

or else

$$
\left|A_{r}\right|>1, \quad\left|B_{k s_{k}}\right|>1 \text { for } k=1,2, \ldots, l .
$$

This theorem is similar to [6, Theorem 4.1], but is stronger in some respects and weaker in others. It is important here that the zeros of $H(z)$ lie in $E$, which we did not have in that theorem. The proof makes use of the following lemma.

Lemma. Suppose that we are given the linear forms

$$
T_{k}=\sum_{j=0}^{l} \alpha_{j k} t_{j} \quad(k=0,1, \ldots, l),
$$

where the coefficients $\alpha_{j k}$ are real, and $\alpha_{j k}>0$ for $j \neq k$. Then there exist positive $t_{0}, t_{1}, \ldots, t_{l}$ for which $T_{0}, T_{1}, \ldots, T_{l}$ all have the same sign (that is, they are all positive, all negative, or all zero).

Proof. The result is trivial if any $\alpha_{j j} \geqq 0$. Suppose, for example, that $\alpha_{00} \geqq 0$. All the forms will be positive at $t_{0}=1, t_{1}=\cdots=t_{l}=0$ if $\alpha_{00}>0$. If $\alpha_{00}=0$, then 
$T_{0}=0$ at this point, but there will be nearby points at which $T_{0}$ as well as the other forms are positive.

Thus we may suppose that all $\alpha_{j j}<0$. The result is clear for $l=0$. Now suppose that $l>0$, and assume the result known for $l-1$. We can add multiples of $T_{l}$ to the other forms to eliminate $t_{l}$, giving

$$
T_{k}+\lambda_{k} T_{l}=\sum_{j=0}^{l-1} \beta_{j k} t_{j} \quad(k=0,1, \ldots, l-1) .
$$

We see that $\lambda_{k}>0$, and $\beta_{j k}>0$ for $j \neq k$. By the inductive hypothesis, we can choose positive $t_{0}, t_{1}, \ldots, t_{l-1}$ so that all $T_{k}+\lambda_{k} T_{l}$ have the same sign. If we choose $t_{l}$ to make $T_{l}=0$, then $t_{l}>0$. Hence we have positive $t_{0}, t_{1}, \ldots, t_{l}$ which make $T_{0}$, $T_{1}, \ldots, T_{l-1}$ have the same sign, and $T_{l}=0$. If all the forms vanish, we are done; otherwise, there will be nearby points where $T_{l}$ has the same sign as the other forms.

Proof of theorem. We may suppose that $F$ contains the points $\infty, p_{1}, \ldots, p_{l}$. We know from Fekete and Szegö [3, Theorem G], that we can find a polynomial

$$
h_{0}(z)=\sum_{\nu=0}^{r^{\prime}} a_{v} z^{v}
$$

with real coefficients, $r^{\prime}>0$ and $a_{r^{\prime}} \neq 0$, and having all of its zeros in $E$, such that $\left|h_{0}(z)\right|<1$ on $E$ and $\left|h_{0}(z)\right|>1$ on $F$. It is not essentially different to find functions

$$
h_{k}(z)=\sum_{\nu=0}^{s_{k}^{\prime}} b_{k v}\left(\frac{q_{k}}{z-p_{k}}\right)^{v} \quad(k=1,2, \ldots, l)
$$

with real coefficients, $s_{k}^{\prime}>0$ and $b_{k s_{k}^{\prime}} \neq 0$, and having all of their zeros in $E$, such that $\left|h_{k}(z)\right|<1$ on $E$ and $\left|h_{k}(z)\right|>1$ on $F$. Putting $p_{0}=\infty$, all of the points $p_{0}, p_{1}, \ldots$, $p_{l}$ are in $F$, and hence $\left|h_{j}\left(p_{k}\right)\right|>1$ for $0 \leqq j \leqq l, 0 \leqq k \leqq l$; of course, there is a pole when $j=k$.

Now put

$$
H(z)=h_{0}(z)^{n_{0}} h_{1}(z)^{n_{1}} \cdots h_{l}(z)^{n_{l}},
$$

where $n_{0}, n_{1}, \ldots, n_{l}$ are positive integers which will be chosen later. Then $H(z)$ has real coefficients, the zeros of $H(z)$ are in $E$, and $|H(z)|<1$ on $E,|H(z)|>1$ on $F$, as required. We must choose $n_{0}, n_{1}, \ldots, n_{l}$ so as to make all the leading coefficients be less than 1 in absolute value, or all greater.

We see that $r=n_{0} r^{\prime}, s_{k}=n_{k} s_{k}^{\prime}$ for $k=1,2, \ldots, l$, and

$$
A_{r}=a_{r^{\prime}}^{n_{0}} h_{1}(\infty)^{n_{1}} \cdots h_{l}(\infty)^{n_{l}}, \quad B_{k s_{k}}=b_{k s_{k}^{\prime}}^{n_{k}} \prod_{j \neq k} h_{j}\left(p_{k}\right)^{n_{j}}
$$

Thus $r>0$ and $A_{r} \neq 0, s_{k}>0$ and $B_{k s_{k}} \neq 0$ for $k=1,2, \ldots, l$. If we put

$$
\begin{aligned}
& \alpha_{00}=\log \left|a_{r^{\prime}}\right|, \quad \alpha_{k k}=\log \left|b_{k s_{k}}\right| \quad \text { for } 1 \leqq k \leqq l, \\
& \alpha_{j k}=\log \left|h_{j}\left(p_{k}\right)\right| \quad \text { for } 0 \leqq j \leqq l, \quad 0 \leqq k \leqq l, j \neq k,
\end{aligned}
$$


then

$$
\begin{aligned}
\log \left|A_{r}\right| & =\sum_{j=0}^{l} \alpha_{j 0} n_{j}, \\
\log \left|B_{k s_{k}}\right| & =\sum_{j=0}^{l} \alpha_{j k} n_{j} \text { for } k=1,2, \ldots, l .
\end{aligned}
$$

We want to choose positive integers $n_{0}, n_{1}, \cdots, n_{l}$ which will make all of these forms positive, or all negative.

Now $\alpha_{j k}>0$ for $j \neq k$, hence, by the lemma, we can find real positive $t_{0}, t_{1}, \ldots, t_{l}$ which make all the forms $\sum \alpha_{j k} t_{j}$ have the same sign. If they are all positive, we can find a nearby rational point at which all are still positive, and can take $n_{0}, n_{1}, \ldots, n_{l}$ proportional to the coordinates of this point. We can proceed in a similar way if all the forms are negative. Finally, if all the forms vanish at a point, we can multiply $h_{0}(z)$ by a factor slightly greater than 1 , which will add a small quantity to all the $\alpha_{0 k}$, and hence make all the forms positive at the point, thus reducing this case to the first case.

5. Proof of the converse. The main theorem asserts that if there is a function $h(z)$ of a certain form with $|h(z)|<1$ on $E$, then any function $f(z)$ satisfying the stated conditions is rational. We wish to show that if no such $h(z)$ exists, then nondenumerably many nonrational functions $f(z)$ can be found. It will be sufficient here to assume that $p_{1}, \ldots, p_{l}, q_{1}, \ldots, q_{l}$ are real, with $p_{j} \neq p_{k}$ for $j \neq k$ and $q_{k} \neq 0$.

Suppose that there is no such $h(z)$. Let $F$ be a closed set contained in $G$ and having $\infty, p_{1}, \ldots, p_{l}$ as inner points. Applying $\S 4$, we find that there exists a function

$$
H(z)=\sum_{v=0}^{r} A_{v} z^{v}+\sum_{k=1}^{l} \sum_{v=1}^{s_{k}} B_{k v}\left(\frac{q_{k}}{z-p_{k}}\right)^{v}
$$

whose zeros lie in $E$, and which has real coefficients with $r>0$ and $0<\left|A_{r}\right|<1$, $s_{k}>0$ and $0<\left|B_{k s_{k}}\right|<1$ for $k=1,2, \ldots, l$, such that $|H(z)|<1$ on $E$ and $|H(z)|>1$ on $F$. We can choose $R>1$ so that $|H(z)| \geqq R$ on $F$. We see that

$$
\begin{aligned}
& \frac{1}{H(z)}=\frac{1}{A_{r}} \cdot \frac{1}{z^{r}}+\cdots \quad \text { near } \infty \\
& \frac{1}{H(z)}=\frac{1}{B_{k s_{k}}}\left(\frac{z-p_{k}}{q_{k}}\right)^{s_{k}}+\cdots \text { near } p_{k} \quad(k=1,2, \ldots, l) .
\end{aligned}
$$

Here the leading coefficients are greater than 1 in absolute value.

If we are given any function regular at $\infty, p_{1}, \ldots, p_{l}$, we can add constant multiples of

$$
\begin{gathered}
z^{r} H(z)^{-n}, z^{r-1} H(z)^{-n}, \ldots, z H(z)^{-n} \\
\left(\frac{q_{k}}{z-p_{k}}\right)^{s_{k}} H(z)^{-n},\left(\frac{q_{k}}{z-p_{k}}\right)^{s_{k}-1} H(z)^{-n}, \ldots, \frac{q_{k}}{z-p_{k}} H(z)^{-n} \quad(k=1,2, \ldots, l)
\end{gathered}
$$


in turn, so as to make the coefficients of

$$
\begin{gathered}
\left(\frac{1}{z}\right)^{n r-r},\left(\frac{1}{z}\right)^{n r-r+1}, \ldots,\left(\frac{1}{z}\right)^{n r-1}, \\
\left(\frac{z-p_{k}}{q_{k}}\right)^{n s_{k}-s_{k}},\left(\frac{z-p_{k}}{q_{k}}\right)^{n s_{k}-s_{k}+1}, \ldots,\left(\frac{z-p_{k}}{q_{k}}\right)^{n s_{k}-1} \quad(k=1,2, \ldots, l)
\end{gathered}
$$

in the expansions at $\infty$ and $p_{k}$ be integers, with at least two choices for each multiplier in $[-1,1]$. The sum of all these terms may be written in the form

$$
g_{n}(z)=\frac{1}{H(z)^{n}}\left\{\sum_{\nu=0}^{r-1} \alpha_{n v} z^{r-v}+\sum_{k=1}^{l} \sum_{v=0}^{s_{k}-1} \beta_{n k v}\left(\frac{q_{k}}{z-p_{k}}\right)^{s_{k}-\nu}\right\}
$$

where $\left|\alpha_{n v}\right| \leqq 1$ and $\left|\beta_{n k v}\right| \leqq 1$. This function is regular at $\infty$ and $p_{k}$ for any $n \geqq 1$, and hence is regular except at the zeros of $H(z)$, which lie in $E$. Now the maximum of $\left|g_{n}(z)\right|$ for $\mid H(z) \geqq R$ occurs for $|H(z)|=R$. Hence if we put

then

$$
K=\max _{|H(z)|=R}\left\{\sum_{\nu=0}^{r-1}|z|^{r-\nu}+\sum_{k=1}^{l} \sum_{\nu=0}^{s_{k}-1}\left|\frac{q_{k}}{z-p_{k}}\right|^{s_{k}-\nu}\right\},
$$

$$
\left|g_{n}(z)\right| \leqq K / R^{n} \text { for }|H(z)| \geqq R .
$$

This inequality therefore holds throughout $F$.

Thus any series of the form

$$
f(z)=\sum_{n=1}^{\infty} g_{n}(z)
$$

will converge uniformly on $F$, and hence $f(z)$ will be regular at inner points of $F$. It is assumed that we choose $g_{1}(z), g_{2}(z), g_{3}(z), \ldots$ in turn by the above procedure, using each to modify the sum of the preceding functions. This will make all the coefficients of $f(z)$ be integers in the usual expansions at $\infty, p_{1}, \ldots, p_{l}$. We find in this way a nondenumerable infinity of functions $f(z)$ with the required expansions and regular at the inner points of $F$. We need to modify the procedure to obtain functions $f(z)$ regular at all points of $G$.

For this purpose, choose an expanding sequence of closed subsets $F_{0}, F_{1}$, $F_{2}, \ldots$ of $G$, such that every point of $G$ is an inner point of some of the sets, and all of them have $\infty, p_{1}, \ldots, p_{l}$ as inner points. Let $H_{m}(z), r_{m}, s_{m k}, R_{m}, g_{m n}(z), K_{m}$ have the same relation to $F_{m}$ as $H(z), r, s_{k}, R, g_{n}(z), K$ have to $F$ above. Then

$$
\left|g_{m n}(z)\right| \leqq K_{m} / R_{m}^{n} \text { on } F_{m} \text {. }
$$

Given any function regular at $\infty, p_{1}, \ldots, p_{l}$, we can add on a function $g_{m n}(z)$ so as to make the coefficients of

$$
\begin{gathered}
\left(\frac{1}{z}\right)^{n r_{m}-r_{m}},\left(\frac{1}{z}\right)^{n r_{m}-r_{m}+1}, \ldots,\left(\frac{1}{z}\right)^{n r_{m}-1}, \\
\left(\frac{z-p_{k}}{q_{k}}\right)^{n s_{m k}-s_{m k}},\left(\frac{z-p_{k}}{q_{k}}\right)^{n s_{m k}-s_{m k}+1}, \ldots,\left(\frac{z-p_{k}}{q_{k}}\right)^{n s_{m k}-1} \quad(k=1,2, \ldots, l)
\end{gathered}
$$


in the expansions at $\infty$ and $p_{k}$ be integers, and indeed with two choices for each coefficient.

If we summed $g_{m n}(z)$ over $n$ for a fixed $m$, we would again obtain a function which might not be regular in all of $G$. Instead of doing this, form a partial sum

$$
f_{m}(z)=\sum_{n=\kappa_{m}+1}^{\lambda_{m}} g_{m n}(z),
$$

where $\kappa_{m}$ and $\lambda_{m}$ will be chosen later. If we are given any function regular at $\infty, p_{1}, \ldots, p_{l}$, we can add on a function of this type which will make the coefficients of

$$
\begin{gathered}
\left(\frac{1}{z}\right)^{\kappa_{m} r_{m}},\left(\frac{1}{z}\right)^{\kappa_{m} r_{m}+1}, \ldots,\left(\frac{1}{z}\right)^{\lambda_{m} r_{m}-1}, \\
\left(\frac{z-p_{k}}{q_{k}}\right)^{\kappa_{m} s_{m k}},\left(\frac{z-p_{k}}{q_{k}}\right)^{\kappa_{m} s_{m k}+1}, \ldots,\left(\frac{z-p_{k}}{q_{k}}\right)^{\lambda_{m} s_{m k}-1} \quad(k=1,2, \ldots, l)
\end{gathered}
$$

in the expansions at $\infty$ and $p_{k}$ be integers, and indeed with two choices for each coefficient.

What we want to do is to choose $\kappa_{m}$ and $\lambda_{m}$ so that we can construct functions $f(z)$ of the form

$$
f(z)=\sum_{m=0}^{\infty} f_{m}(z)=\sum_{m=0}^{\infty} \sum_{n=\kappa_{m}+1}^{\lambda_{m}} g_{m n}(z)
$$

which will be regular in $G$ and have integer coefficients in the expansions at $\infty$, $p_{1}, \ldots, p_{l}$.

We can make all the coefficients be integers by choosing the $f_{m}(z)$ in turn, provided that

$$
\begin{aligned}
\kappa_{0} & =0, & & \\
\kappa_{m+1} r_{m+1} & \leqq \lambda_{m} r_{m} & & \text { for } m \geqq 0, \\
\kappa_{m+1} s_{m+1, k} & \leqq \lambda_{m} s_{m k} & & \text { for } 1 \leqq k \leqq l \text { and } m \geqq 0, \\
\lambda_{m} & \rightarrow \infty & & \text { as } m \rightarrow \infty .
\end{aligned}
$$

These conditions guarantee that we can start, continue without a gap, and get indefinitely far. As before, there will be nondenumerably many choices.

On the other hand,

$$
\left|f_{m}(z)\right| \leqq \sum_{n=\kappa_{m}+1}^{\lambda_{m}} \frac{K_{m}}{R_{m}^{n}}<\frac{K_{m}}{R_{m}^{\kappa_{m}}\left(R_{m}-1\right)}
$$

on $F_{m}$. We must have $\kappa_{0}=0$, but for $m>0$, we can choose $\kappa_{m}$ so that

$$
R_{m}^{x_{m}}\left(R_{m}-1\right)>2^{m} K_{m}
$$

Then $\left|f_{m}(z)\right|<1 / 2^{m}$ on $F_{m}$ for $m>0$, and hence the series $f(z)=\sum f_{m}(z)$ will converge uniformly in the interior of $G$, no matter how the $\lambda_{m}$ are chosen. We can then choose 
the $\lambda_{m}$ so that the conditions relating $\kappa_{0}, \kappa_{1}, \kappa_{2}, \ldots, \lambda_{0}, \lambda_{1}, \lambda_{2}, \ldots$ are satisfied. In this way, we find a nondenumerable infinity of functions $f(z)$ which are regular in $G$ and have integer coefficients in all the expansions.

It is easily seen that a rational function whose expansion at $\infty$ has rational coefficients is expressible as the quotient of two polynomials with rational coefficients. Thus only a denumerable infinity of the functions $f(z)$ which we have constructed can be rational. We have therefore found a nondenumerable infinity of nonrational functions which are regular in $G$ and have the required expansions with integer coefficients.

\section{REFERENCES}

1. F. Carlson, Uber Potenzreihen mit ganzzahligen Koeffizienten, Math. Z. 9 (1921), 1-13.

2. M. Fekete, Über die Verteilung der Wurzeln bei gewissen algebraischen Gleichungen mit ganzzahligen Koeffizienten, Math. Z. 17 (1923), 228-249.

3. M. Fekete and G. Szegö, On algebraic equations with integral coefficients whose roots belong to a given point set, Math. Z. 63 (1955), 158-172.

4. G. Pólya, Sur les séries entières à coefficients entiers, Proc. London Math. Soc. (2) 21 (1922), 22-38.

5. - Über gewisse notwendige Determinantenkriterien für die Fortsetzbarkeit einer Potentzreihe, Math. Ann. 99 (1928), 687-706.

6. R. M. Robinson, On the distribution of certain algebraic integers, Math. Z. 99 (1967), $28-41$.

UNIVERSITY OF CALIFORNIA,

Berkeley, CALIFORNIA 